\title{
A Bayesian Filter for Sound Environment System with Quantized Observation*
}

\author{
Hisako Orimoto, Akira Ikuta \\ Department of Management Information Systems, Prefectural University of Hiroshima, Hiroshima, Japan \\ Email: orimoto@pu-hiroshima.ac.jp
}

How to cite this paper: Orimoto, $\mathrm{H}$. and Ikuta, A. (2018) A Bayesian Filter for Sound Environment System with Quantized Observation. Intelligent Information Management, 10, 87-98. https://doi.org/10.4236/iim.2018.103007

Received: April 23, 2018

Accepted: May 22, 2018

Published: May 25, 2018

Copyright (C) 2018 by authors and Scientific Research Publishing Inc. This work is licensed under the Creative Commons Attribution International License (CC BY 4.0).

http://creativecommons.org/licenses/by/4.0/

(c) (i) Open Access

\begin{abstract}
In the real sound environment, the observation data are usually contaminated by additional background noise of arbitrary distribution type. In order to estimate several evaluation quantities for specific signal based on the observed noisy data, it is fundamental to estimate the fluctuating wave form of the specific signal. On the other hand, the observation data are very often measured in a digital level form at discrete times. This is because some signal processing methods by utilizing a digital computer are indispensable for extracting exactly various kinds of statistical evaluation for the specific signal based on the quantized level data. In this study, a Bayesian filter matched to the complicated sound environment system is derived. First, in the real situation where the sound environment system is affected by background noise of arbitrary probability distribution, a stochastic system model with quantized observation is established. Next, two types of the recursive algorithm of Bayesian filter to estimate the unknown specific signal are theoretically proposed in the quantized level form. Finally, the effectiveness of the proposed theory is experimentally confirmed by applying it to the estimation problem of real sound environment.
\end{abstract}

\section{Keywords}

Bayesian Filter, Sound Environment, Quantized Observation

\section{Introduction}

In the real sound environment, the observation data are usually contaminated by additional external noise (i.e., background noise) of arbitrary distribution type. In order to estimate several evaluation quantities for specific signal, like $L_{x}$

${ }^{*}$ New type method is proposed based on the Baysian filter by use of the quantized observation in sound environment system. 
$\left((100-x)\right.$ percentile level), $L_{e q}$ (averaged energy on decibel scale) and peak value, based on the observed noisy data, it is fundamental to estimate the momentarily fluctuating wave form of the specific signal.

Up to now, many methodological studies have been reported on the state estimation for stochastic systems [1] [2] [3]. However, many standard estimation methods proposed previously in a study of stochastic systems are restricted only to the Gaussian distribution [4] [5]. Several state estimation methods for nonlinear system have been also proposed by assuming the Gaussian distribution of system and observation noises [6] [7] [8] [9] [10]. The real sound environment often shows an intricate fluctuation pattern rather than the standard Gaussian distribution. In our previous studies [11] [12] [13] [14] [15], several state estimation methods for a sound environment system with non-Gaussian fluctuations have been proposed on the basis of expansion expressions for the probability distribution. Furthermore, state estimation methods for stochastic systems with complex characteristics and/or unknown structure have been proposed by using Bayes theorem on probability distribution [16] [17] [18]. Especially, though the unscented Kalman filter (UKF) and particle filter are useful for non-linear systems, UKF considers only the mean and variance of variables, and the particle filter needs very complicated algorithm based on Monte Carlo simulation [10] [18].

On the other hand, in the actual case contaminated by the background noise, some signal processing methods utilizing a digital computer are indispensable for estimating precisely the latent specific signal based on the noisy observations. Therefore, the observed data in an analogue form have to be transformed into a digital one at discrete time. However, many standard estimation methods proposed previously in stochastic systems are restricted only to a continuous level form of the observation.

In this study, a state estimation algorithm of an object-oriented type matched to the above real problem and the computer technique with successive observation for the complicated sound environment system is derived. First, in the real situation where the sound environment system is affected by background noise of arbitrary probability distribution, a stochastic process model with a quantized observation is established in Section 2. Next, by considering the linear and higher order correlation information between the specific signal and observation, two types of the recursive algorithms of Bayesian filter to estimate the unknown specific signal are theoretically proposed in the quantized level form matched to the signal processing by use of a digital computer. More specifically, in Section 3.1, an estimation algorithm in functional forms is derived by introducing the forward difference operator instead of usual differential operation. Furthermore, in Section 3.2, a method combining the analytical formula with Monte Carlo simulation is derived by introducing the idea of particles. Finally, the effectiveness of the proposed theory is experimentally confirmed too by applying it to the estimation problem of real sound environment in Section 4. 


\section{Formulation of Sound Environment System with Quantized Observation}

Let us consider an arbitrary stochastic environment system with the power state variables of arbitrary distribution type, and express the system equation as:

$$
x_{k+1}=F x_{k}+G u_{k}
$$

where $x_{k}$ denotes the specific signal at a discrete time $k, u_{k}$ is the random input with known statistics. Here, $x_{k}$ and $u_{k}$ are statistically independent of each other. Two parameters $F$ and $G$ are estimated by using an auto-correlation technique [1]. Furthermore, the observation model is established by considering the additive property of power variables and the quantized observation in decibel scale, as follows:

$$
\begin{gathered}
y_{k}=10 \log _{10}\left\{\left(x_{k}+v_{k}\right) / 10^{-12}\right\} \\
z_{k}=Q\left(y_{k}\right) \equiv g\left(x_{k}+v_{k}\right)
\end{gathered}
$$

where $y_{k}$ is the noisy observation contaminated by the additive background noise $v_{k}$. Though $y_{k}$ is decibel variable with continuous level, the observation data are measured in a quantized level form suitable for the signal processing by use of a digital computer through A/D converter. The function $Q\left(y_{k}\right)$ denotes a nonlinear function expressing the quantization mechanism and $z_{k}$ is the quantized observation. Therefore, $g(v)$ denotes a nonlinear function combining the nonlinearity of decibel observation with the quantized observation mechanism. In this study, a Bayesian filter to estimate the specific signal $x_{k}$ is proposed on the basis of the quantized observation contaminated by the background noise $v_{k}$.

\section{Establishment of Bayesian Filter with Quantized Observation}

\subsection{General Expression of Bayesian Filter in Expansion Series Form}

In order to express explicitly the effect of successive observation $z_{k}$ on the estimated probability density function $P\left(x_{k} \mid Z_{k}\right)$ by use of various types of linear and/or nonlinear correlation between $x_{k}$ and $z_{k}$, the well-known Bayes' theorem is introduced.

$$
P\left(x_{k} \mid Z_{k}\right)=P\left(x_{k}, z_{k} \mid Z_{k-1}\right) / P\left(z_{k} \mid Z_{k-1}\right)
$$

where $\left(Z_{k} \equiv\left\{z_{1}, z_{2}, \cdots, z_{k}\right\}\right)$ is a set of observation data up to time $k$. By expanding the conditional probability density function $P\left(x_{k} \mid Z_{k}\right)$ in a statistical orthogonal expansion series on the basis of the well-known standard probability distributions describing the dominant part of the actual fluctuation, the following expression is derived [11] [12].

$$
P\left(x_{k} \mid Z_{k}\right)=\frac{P_{0}\left(x_{k} \mid Z_{k-1}\right) \sum_{m=0}^{\infty} \sum_{n=0}^{\infty} A_{m n} \phi_{m}^{(1)}\left(x_{k}\right) \phi_{n}^{(2)}\left(z_{k}\right)}{\sum_{n=0}^{\infty} A_{0 n} \phi_{n}^{(2)}\left(z_{k}\right)}
$$


with

$$
A_{m n} \equiv\left\langle\phi_{m}^{(1)}\left(x_{k}\right) \phi_{n}^{(2)}\left(z_{k}\right) \mid Z_{k-1}\right\rangle
$$

The above two functions $\phi_{m}^{(1)}\left(x_{k}\right)$ and $\phi_{n}^{(2)}\left(z_{k}\right)$ are orthonormal polynomials of degrees $m$ and $n$ with weighting functions $P_{0}\left(x_{k} \mid Z_{k-1}\right)$ and $P_{0}\left(z_{k} \mid Z_{k-1}\right)$. Based on Equation (5), the estimate of the polynomial function $f_{M}\left(x_{k}\right)$ of $x_{k}$ with $M$ th order can be derived as follows.

$$
\begin{aligned}
\hat{f}_{M}\left(x_{k}\right) & \equiv\left\langle f_{M}\left(x_{k}\right) \mid Z_{k}\right\rangle \\
& =\sum_{m=0}^{M} \sum_{n=0}^{\infty} C_{M m} A_{m n} \phi_{n}^{(2)}\left(z_{k}\right) / \sum_{n=0}^{\infty} A_{0 n} \phi_{n}^{(2)}\left(z_{k}\right)
\end{aligned}
$$

where $C_{M m}$ is an appropriate constant satisfying the following equality:

$$
f_{M}\left(x_{k}\right)=\sum_{m=0}^{M} C_{M m} \phi_{m}^{(1)}\left(x_{k}\right)
$$

\subsection{Estimation Algorithm by Introducing Difference Operation}

In order to make the general theory for estimation algorithm more concrete, Gaussian distribution is considered as an example of standard probability functions for the specific signal:

$$
\begin{gathered}
P_{0}\left(x_{k} \mid Z_{k-1}\right)=N\left(x_{k} ; x_{k}^{*}, \Gamma_{x_{k}}\right) \\
N\left(x ; \mu, \sigma^{2}\right) \equiv \frac{1}{\sqrt{2 \pi \sigma^{2}}} \exp \left\{-\frac{(x-\mu)^{2}}{2 \sigma^{2}}\right\}, \\
x_{k}^{*} \equiv\left\langle x_{k} \mid Z_{k-1}\right\rangle, \Gamma_{x_{k}} \equiv\left\langle\left(x_{k}-x_{k}^{*}\right)^{2} \mid Z_{k-1}\right\rangle
\end{gathered}
$$

Furthermore, as the fundamental probability function on the level-quantized observation, the generalized binomial distribution [19] with level difference interval $h_{z}$ can be chosen:

$$
\begin{gathered}
P_{0}\left(z_{k} \mid Z_{k-1}\right)=\frac{\left(\frac{N_{k}-z_{M}}{h_{z}}\right) !}{\left(\frac{z_{k}-z_{M}}{h_{z}}\right) !\left(\frac{\left.N_{k}-z_{k}\right)}{h_{z}}\right) !} p_{k}^{\frac{z_{k}-z_{M}}{h_{z}}}\left(1-p_{k}\right)^{\frac{N_{k}-z_{k}}{h_{z}}} \\
p_{k} \equiv \frac{z_{k}^{*}-z_{M}}{N_{k}-z_{M}}, N_{k} \equiv \frac{\left(z_{k}^{*}-z_{M}\right) h_{z} z_{k}^{*}-z_{M} \Omega_{z_{k}}}{\left(z_{k}^{*}-z_{M}\right) h_{z}-\Omega_{z_{k}}} \\
z_{k}^{*} \equiv\left\langle z_{k} \mid Z_{k-1}\right\rangle, \Omega_{z_{k}} \equiv\left\langle\left(z_{k}-z_{k}^{*}\right)^{2} \mid Z_{k-1}\right\rangle
\end{gathered}
$$

where $z_{M}$ is the minimum level of observations. The orthonormal polynomials with two weighting probability distributions in Equations (9) and (10) can be determined as

$$
\phi_{m}^{(1)}\left(x_{k}\right)=\frac{1}{\sqrt{m !}} H_{m}\left(\frac{x_{k}-x_{k}^{*}}{\sqrt{\Gamma_{x_{k}}}}\right)
$$




$$
\begin{aligned}
\phi_{n}^{(2)}\left(z_{k}\right)= & \left\{\left(\frac{N_{k}-z_{M}}{h_{z}}\right)^{(n)} n !\right\}^{-1 / 2}\left(\frac{1-p_{k}}{p_{k}}\right)^{n / 2} \frac{1}{h_{z}^{n}} \\
& \cdot \sum_{j=0}^{n}\left(\begin{array}{l}
n \\
j
\end{array}\right)(-1)^{n-j}\left(\frac{p_{k}}{1-p_{k}}\right)^{n-j}\left(N_{k}-z_{k}\right)^{(n-j)}\left(z_{k}-z_{M}\right)^{(j)}
\end{aligned}
$$

where $H_{m}(\cdot)$ denotes the Hermite polynomial with $m$ th order, and $z^{(j)}$ is the th order factorial function defined by [19]

$$
z^{(n)}=z\left(z-h_{z}\right)\left(z-2 h_{z}\right) \cdots\left(z-(n-1) h_{z}\right), z^{(0)}=1
$$

Since the function $g(\cdot)$ in Equation (3) is not differentiable in general, the following expansion expression of discrete type is introduced with two arbitrary constants $d_{v}$ and $h_{v}$.

$$
g(x+v)=\sum_{n=0}^{\infty} \frac{1}{n !}\left(\frac{d_{v}}{h_{v}}\right)^{n} x^{(n)} \Delta^{n} g(v)
$$

where $\Delta$ is the forward difference operator defined as:

$$
\Delta g(v) \equiv \frac{1}{d_{v}}\left\{g\left(v+h_{v}\right)-g(v)\right\}
$$

After substituting Equations (2) and (3) into the definition of two parameters of $z_{k}$ and $\Omega_{z_{k}}$ in Equation (10), by applying Equation (14), the following expressions can be derived (in the case of $d_{v}=h_{v}$ ).

$$
\begin{aligned}
z_{k}^{*} & =\left\langle g\left(x_{k}+v_{k}\right) \mid Z_{k-1}\right\rangle \\
& =\left\langle g\left(v_{k}\right)+x_{k} \Delta g\left(v_{k}\right)+\frac{1}{2} x_{k}\left(x_{k}-h_{v}\right) \Delta^{2} g\left(v_{k}\right)+\cdots \mid Z_{k-1}\right\rangle \\
\Omega_{z_{k}}= & \left\langle\left\{g\left(x_{k}+v_{k}\right)-z_{k}^{*}\right\}^{2} \mid Z_{k-1}\right\rangle \\
= & \left\langle\left\{g\left(v_{k}\right)+x_{k} \Delta g\left(v_{k}\right)+\frac{1}{2} x_{k}\left(x_{k}-h_{v}\right) \Delta^{2} g\left(v_{k}\right)+\cdots\right\}^{2} \mid Z_{k-1}\right\rangle-z_{k}^{*}
\end{aligned}
$$

Furthermore, the expansion coefficients defined by Equation (6) can be expressed as follows:

$$
\begin{aligned}
A_{m n}= & \left\{\left(\frac{N_{k}-z_{M}}{h_{z}}\right)^{(n)} m ! n !\right\}^{-1 / 2}\left(\frac{1-p_{k}}{p_{k}}\right)^{n / 2} \frac{1}{h_{z}^{n}} \sum_{j=0}^{n}\left(\begin{array}{c}
n \\
j
\end{array}\right)(-1)^{n-j}\left(\frac{p_{k}}{1-p_{k}}\right)^{n-j} \\
& \cdot\left\langle H_{m}\left(\frac{x_{k}-x_{k}^{*}}{\sqrt{\Gamma_{x_{k}}}}\right)\left(N_{k}-g\left(x_{k}+v_{k}\right)\right)^{(n-j)}\left(g\left(x_{k}+v_{k}\right)-z_{M}\right)^{(j)} \mid Z_{k-1}\right\rangle
\end{aligned}
$$

The above Equations (16)-(18) can be obtained from the statistics of the background noise $v_{k}$ and the predictions of $x_{k}$ at a discrete time $k-1$; i.e., the expectation values of arbitrary functions of $x_{k}$ conditioned by $Z_{k-1}$.

Therefore, the estimation algorithm in Equation (7) can be expressed explicitly as follows: 


$$
\begin{aligned}
\hat{f}_{M}\left(x_{k}\right)= & \sum_{m=0}^{M} \sum_{n=0}^{\infty} C_{M m} A_{m n}\left\{\left(\frac{N_{k}-z_{M}}{h_{z}}\right)^{(n)} n !\right\}^{-1 / 2}\left(\frac{1-p_{k}}{p_{k}}\right)^{n / 2} \frac{1}{h_{z}^{n}} \\
& \cdot \sum_{j=0}^{n}\left(\begin{array}{l}
n \\
j
\end{array}\right)(-1)^{n-j}\left(\frac{p_{k}}{1-p_{k}}\right)^{n-j}\left(N_{k}-z_{k}\right)^{(n-j)}\left(z_{k}-z_{M}\right)^{(j)} / I
\end{aligned}
$$

with

$$
\begin{aligned}
I= & \sum_{n=0}^{\infty} A_{0 n}\left\{\left(\frac{N_{k}-z_{M}}{h_{z}}\right)^{(n)} n !\right\}^{-1 / 2}\left(\frac{1-p_{k}}{p_{k}}\right)^{n / 2} \frac{1}{h_{z}^{n}} \\
& \cdot \sum_{j=0}^{n}\left(\begin{array}{l}
n \\
j
\end{array}\right)(-1)^{n-j}\left(\frac{p_{k}}{1-p_{k}}\right)^{n-j}\left(N_{k}-z_{k}\right)^{(n-j)}\left(z_{k}-z_{M}\right)^{(j)}
\end{aligned}
$$

Especially, the estimates for mean and variance can be obtained as follows:

$$
\begin{aligned}
& \hat{x}_{k} \equiv\left\langle x_{k} \mid Z_{k}\right\rangle \\
&= \sum_{m=0}^{1} \sum_{n=0}^{\infty} C_{1 m} A_{m n}\left\{\left(\frac{N_{k}-z_{M}}{h_{z}}\right)^{(n)} n !\right\}\left(\frac{1-p_{k}}{p_{k}}\right)^{n / 2} \frac{1}{h_{z}^{n}} \\
& \cdot \sum_{j=0}^{n}\left(\begin{array}{c}
n \\
j
\end{array}\right)(-1)^{n-j}\left(\frac{p_{k}}{1-p_{k}}\right)^{n-j}\left(N_{k}-z_{k}\right)^{(n-j)}\left(z_{k}-z_{M}\right)^{(j)} / I \\
&\left(C_{10}=x_{k}^{*}, C_{11}=\sqrt{\Gamma_{x_{k}}}\right) \\
& P_{k} \equiv\left\langle\left(x_{k}-\hat{x}_{k}\right)^{2} \mid Z_{k}\right\rangle \sum_{m=0}^{2} \sum_{n=0}^{\infty} C_{2 m} A_{m n}\left\{\left(\frac{N_{k}-z_{M}}{h_{z}}\right)^{(n)} n !\right\}^{-1 / 2}\left(\frac{1-p_{k}}{p_{k}}\right)^{n / 2} \frac{1}{h_{z}^{n}} \\
& \cdot \sum_{j=0}^{n}\left(\begin{array}{l}
n \\
j
\end{array}\right)(-1)^{n-j}\left(\frac{p_{k}}{1-p_{k}}\right)^{n-j}\left(N_{k}-z_{k}\right)^{(n-j)}\left(z_{k}-z_{M}\right)^{(j)} / I \\
&\left(C_{20}=\Gamma_{x_{k}}+\left(x_{k}^{*}-\hat{x}_{k}\right)^{2}, C_{21}=2 \sqrt{\Gamma_{x_{k}}}\left(x_{k}^{*}-\hat{x}_{k}\right), C_{22}=\sqrt{2} \Gamma_{x_{k}}\right)
\end{aligned}
$$

\subsection{Estimation Algorithm by Introducing Particles}

Though the particle filter is useful for the state estimation problem of non-linear systems, this filter needs very complicated algorithm and a large number of computational times based on Monte Carlo simulation and the resampling procedure [16]. In this section, a hybrid algorithm combining the analytical formula for state estimation with Monte Carlo simulation by use of particles is proposed.

The well-known Gaussian distribution is adopted as $P_{0}\left(x_{k} \mid Z_{k-1}\right)$ and $P_{0}\left(z_{k} \mid Z_{k-1}\right)$, because this probability density function is the most standard one.

$$
P_{0}\left(x_{k} \mid Z_{k-1}\right)=N\left(x_{k} ; x_{k}^{*}, \Gamma_{x_{k}}\right), \quad P_{0}\left(z_{k} \mid Z_{k-1}\right)=N\left(z_{k} ; z_{k}^{*}, \Omega_{z_{k}}\right)
$$

Then, the orthonormal functions with two weighting probability density functions in (23) can be given in the Hermite polynomial: 


$$
\phi_{m}^{(1)}\left(x_{k}\right)=\frac{1}{\sqrt{m !}} H_{m}\left(\frac{x_{k}-x_{k}^{*}}{\sqrt{\Gamma_{x_{k}}}}\right), \phi_{n}^{(2)}\left(z_{k}\right)=\frac{1}{\sqrt{n !}} H_{n}\left(\frac{z_{k}-z_{k}^{*}}{\sqrt{\Omega_{z_{k}}}}\right)
$$

Accordingly, the estimation algorithm of the specific signal in Equation (7) can be given by

$$
\hat{f}_{M}\left(x_{k}\right)=\sum_{m=0}^{M} \sum_{n=0}^{\infty} C_{M m} A_{m n} \frac{1}{\sqrt{n !}} H_{n}\left(\frac{z_{k}-z_{k}^{*}}{\sqrt{\Omega_{z_{k}}}}\right) / J
$$

with

$$
J=\sum_{n=0}^{\infty} A_{0 n} \frac{1}{\sqrt{n !}} H_{n}\left(\frac{z_{k}-z_{k}^{*}}{\sqrt{\Omega_{z_{k}}}}\right)
$$

Furthermore, the estimates for mean and variance can be obtained as follows:

$$
\begin{aligned}
& \hat{x}_{k}=\sum_{m=0}^{1} \sum_{n=0}^{\infty} C_{1 m} A_{m n} \frac{1}{\sqrt{n !}} H_{n}\left(\frac{z_{k}-z_{k}^{*}}{\sqrt{\Omega_{z_{k}}}}\right) / J \\
& P_{k}=\sum_{m=0}^{2} \sum_{n=0}^{\infty} C_{2 m} A_{m n} \frac{1}{\sqrt{n !}} H_{n}\left(\frac{z_{k}-z_{k}^{*}}{\sqrt{\Omega_{z_{k}}}}\right) / J
\end{aligned}
$$

Thus, two parameters $z_{k}^{*}, \Omega_{z_{k}}$ and the expansion coefficients $A_{m n}$ are expressed as follows:

$$
\begin{gathered}
z_{k}^{*}=\left\langle g\left(x_{k}+v_{k}\right) \mid Z_{k-1}\right\rangle \\
=\iint g\left(x_{k}+v_{k}\right) P\left(x_{k} \mid Z_{k-1}\right) P\left(v_{k}\right) \mathrm{d} x_{k} \mathrm{~d} v_{k} \\
\Omega_{z_{k}}=\left\langle\left(g\left(x_{k}+v_{k}\right)-z_{k}^{*}\right)^{2} \mid Z_{k-1}\right\rangle \\
=\iint\left(g\left(x_{k}+v_{k}\right)-z_{k}^{*}\right)^{2} P\left(x_{k} \mid Z_{k-1}\right) P\left(v_{k}\right) \mathrm{d} x_{k} \mathrm{~d} v_{k} \\
A_{m n}=\left\langle\frac{1}{\sqrt{m !}} H_{m}\left(\frac{x_{k}-x_{k}^{*}}{\sqrt{\Gamma_{x_{k}}}}\right) \frac{1}{\sqrt{n !}} H_{n}\left(\frac{g\left(x_{k}+v_{k}\right)-z_{k}^{*}}{\sqrt{\Omega_{z_{k}}}}\right) \mid Z_{k-1}\right\rangle \\
=\frac{1}{\sqrt{m !}} \frac{1}{\sqrt{n !}} \iint H_{m}\left(\frac{x_{k}-x_{k}^{*}}{\sqrt{\Gamma_{x_{k}}}}\right) H_{n}\left(\frac{g\left(x_{k}+v_{k}\right)-z_{k}^{*}}{\sqrt{\Omega_{z_{k}}}}\right) P\left(x_{k} \mid Z_{k-1}\right) P\left(v_{k}\right) \mathrm{d} x_{k} \mathrm{~d} v_{k}
\end{gathered}
$$

The integrals in Equations (29)-(31) are evaluated by use of particles with mean $x_{k}^{*}$, variance $\Gamma_{x_{k}}$, higher order statistics $A_{m 0}$ and statistics of the background noise.

\subsection{Prediction Algorithm}

By considering Equation (1), the prediction step essential to perform the recurrence estimation can be given by

$$
x_{k+1}^{*} \equiv\left\langle x_{k+1} \mid Z_{k}\right\rangle=F \hat{x}_{k}+G\left\langle u_{k}\right\rangle
$$




$$
\Gamma_{x_{k+1}} \equiv\left\langle\left(x_{k+1}-x_{k+1}^{*}\right)^{2} \mid Z_{k}\right\rangle=F^{2} P_{k}+G^{2}\left\langle\left(u_{k}-\left\langle u_{k}\right\rangle\right)^{2}\right\rangle
$$

By replacing $k$ with $k+1$, the recurrence estimation can be achieved.

\section{Application to Sound Environment}

In order to examine the practical usefulness of the proposed Bayesian filter based on the quantized observation, the proposed method is applied to the actual sound environmental data. The road traffic noise is adopted as an example of a specific signal with a complex fluctuation form. Applying the proposed estimation method to actually observed data contaminated by background noise and quantized with $2 \mathrm{~dB}$ width roughly, the fluctuation wave form of the specific signal is estimated. The statistics of the specific signal and the background noise used in the experiment are shown in Table 1 and Table 2 respectively.

Figure 1 and Figure 2 show the estimation results of the fluctuation wave form of the specific signal for Data 1 and Data 2 by applying the algorithm proposed in Secttion 3.2. In this estimation, the finite number of expansion

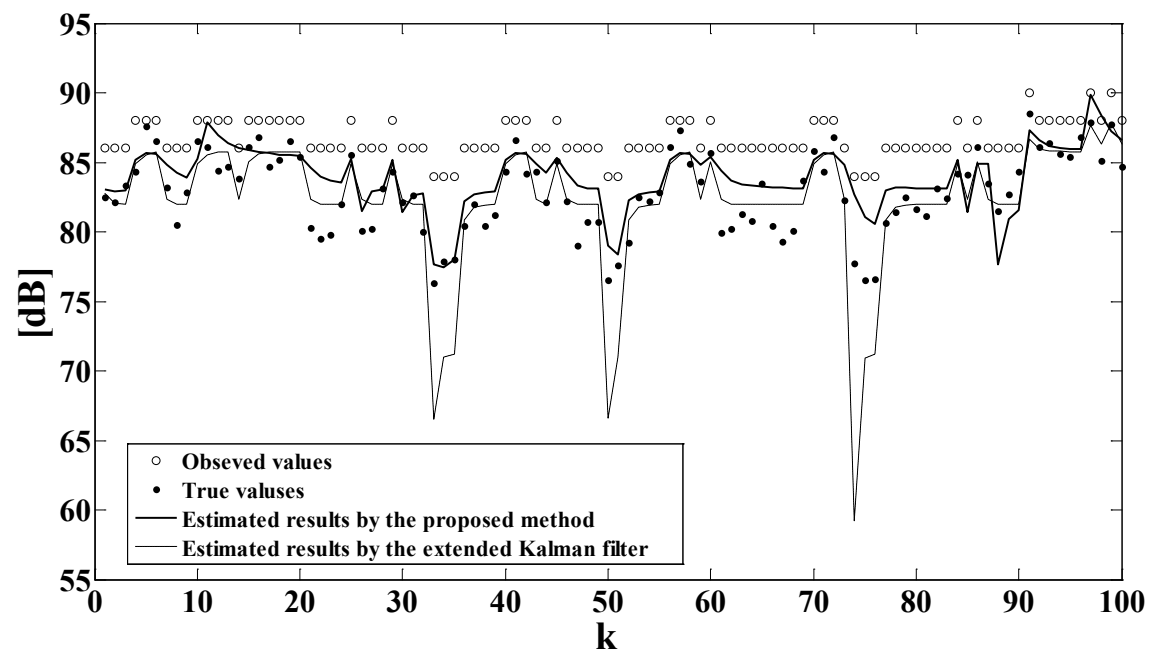

Figure 1. Estimation results for Data 1 of the specific signal by applying the proposed method in Section 3.2 based on the quantized observation data with $2 \mathrm{~dB}$ width.

Table 1. Mean and standard deviation of the specific signal (in $\left.\mathrm{W} / \mathrm{m}^{2}\right)$.

\begin{tabular}{cccccc}
\hline Data & Data 1 & Data 2 & Data 3 & Data 4 & Data 5 \\
\hline Mean Value & $2.23 \times 10^{-4}$ & $3.44 \times 10^{-4}$ & $3.25 \times 10^{-4}$ & $3.82 \times 10^{-4}$ & $3.71 \times 10^{-4}$ \\
Standard Deviation & $1.47 \times 10^{-4}$ & $2.13 \times 10^{-4}$ & $2.65 \times 10^{-4}$ & $3.19 \times 10^{-4}$ & $3.56 \times 10^{-4}$ \\
\hline
\end{tabular}

Table 2. Mean and standard deviation of the background noise (in $\mathrm{W} / \mathrm{m}^{2}$ ).

\begin{tabular}{cccccc}
\hline Data & Data 1 & Data 2 & Data 3 & Data 4 & Data 5 \\
\hline Mean Value & $2.50 \times 10^{-4}$ & $2.49 \times 10^{-4}$ & $2.44 \times 10^{-4}$ & $2.47 \times 10^{-4}$ & $2.48 \times 10^{-4}$ \\
Standard Deviation & $1.08 \times 10^{-5}$ & $9.61 \times 10^{-6}$ & $9.49 \times 10^{-6}$ & $1.05 \times 10^{-5}$ & $9.26 \times 10^{-6}$ \\
\hline
\end{tabular}




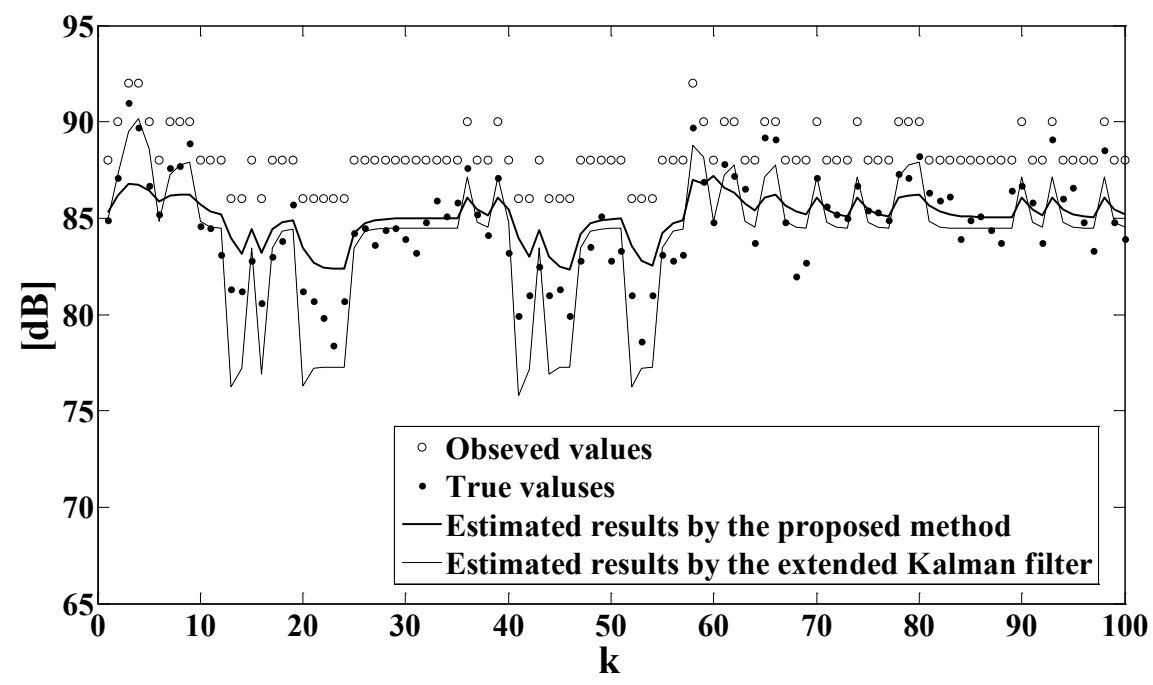

Figure 2. Estimation results for Data 2 of the specific signal by applying the proposed method in Section 3.2 based on the quantized observation data with $2 \mathrm{~dB}$ width.

coefficients $A_{m n}(m, n \leq 2)$ is used for the simplification of the estimation algorithm. In these figures, the horizontal axis shows the discrete time $k$, of the estimation process, and the vertical axis expresses the sound level taking a logarithmic transformation of power-scaled variables, because the actual sound environment usually is evaluated on $\mathrm{dB}$ scale. For comparison, the estimation results calculated by using the usual method are also shown in these figures. Since Kalman's filtering theory is widely used in the field of stochastic system [11] [12], the extended Kalman filter is also applied to the observation data as a trail by introducing the following observation model.

$$
z_{k}=10 \log _{10}\left(x_{k}+v_{k}\right) / 10^{-12}+\varepsilon_{k}
$$

where $\varepsilon_{k}$ denotes the quantized noise and a uniform distribution within $[-q / 2, q / 2]$ ( $q$ : the quantized width) is assumed as the probability distribution of $\varepsilon_{k}$. The results estimated by the proposed method show good agreement with the true values. On the other hand, there are great discrepancies between the estimates based on the standard type dynamical estimation method (i.e., extended Kalman filter), particularly in the estimation of the lower level values of the fluctuation.

Furthermore, the estimation algorithm proposed in Section 3.3 is applied to the observation data. The estimated results of two cases by applying the proposed algorithm to the quantized data with $2 \mathrm{~dB}$ width are shown in Figure 3 and Figure 4 respectively.

The squared sums of the estimation error are shown in Table 3. It can be found numerically that the proposed method is more useful than the extended Kalman filter.

\section{Conclusions}

In this study, state estimation method for sound environment system with 


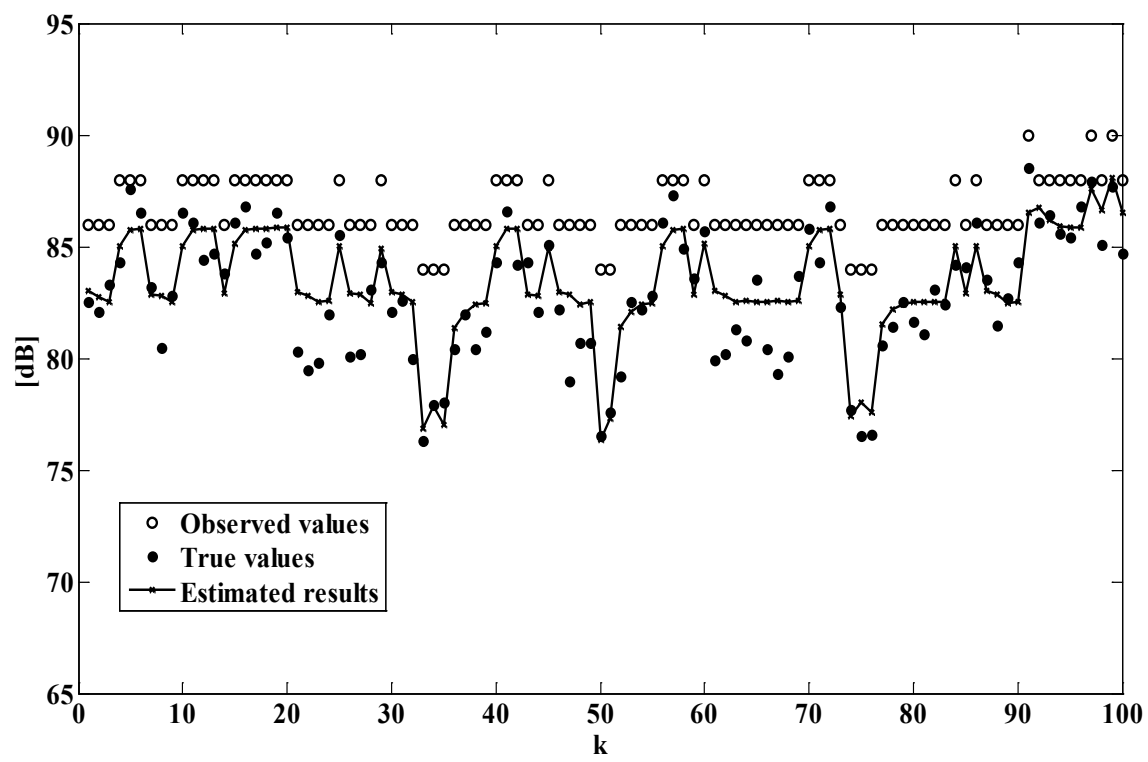

Figure 3. Estimation results for Data 1 of the specific signal by applying the proposed method in Section 3.3 based on the quantized observation data with $2 \mathrm{~dB}$ width.

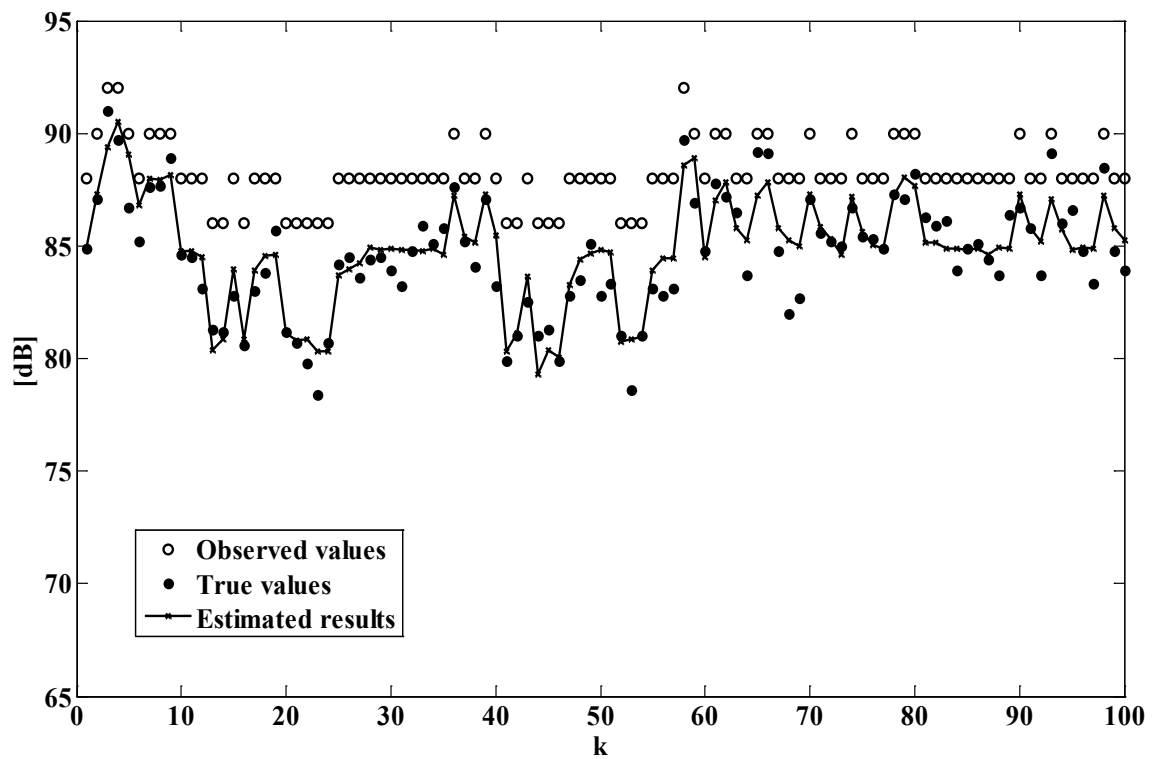

Figure 4. Estimation results for Data 2 of the specific signal by applying the proposed method in Section 3.3 based on the quantized observation data with $2 \mathrm{~dB}$ width.

Table 3. Comparison between the proposed method and the extended Kalman filter for root-mean squared error of the estimation based on the quantized observation data with $2 \mathrm{~dB}$ width (in $\mathrm{dB}$ ).

\begin{tabular}{cccccc}
\hline Data & Data 1 & Data 2 & Data 3 & Data 4 & Data 5 \\
\hline Proposed Method in Section 3.2 & 2.06 & 1.73 & 2.14 & 2.08 & 2.67 \\
Proposed Method in Section 3.3 & 1.39 & 1.09 & 1.81 & 1.87 & 2.24 \\
Extended Kalman Filter & 2.96 & 1.75 & 1.88 & 2.23 & 3.36 \\
\hline
\end{tabular}


quantized level observation has been theoretically proposed on the basis of Bayes' theorem. More specifically, two types of the recursive algorithm of Bayesian filter to estimate the specific unknown signal have been derived based on the quantized level observation matched for the signal processing by use of a digital computer. Furthermore, the validity and effectiveness of the proposed theory have been experimentally confirmed by applying it to the real environmental noise data in sound environment.

The proposed approach is still at the early of study, and there are left a number of practical problems to be continued in the future. For example, the proposed method has to be applied to many other actual data of sound environment. Furthermore, the proposed theory has to be extended to more complicated situations involving multi-signal sources, and an optimal number of expansion terms in the proposed estimation algorithm of expansion type has to be found.

\section{Acknowledgements}

The author is grateful for valuable suggestions at Inter-Noise 2016 [20]. This work was supported in part by fund from the Grant-in-Aid for Scientific Research No.15K06116 from the Ministry of Education, Culture, Sports, Science and Technology-Japan.

\section{References}

[1] Eykhoff, P. (1974) System Identification: Parameter and State Estimation. Wiley, New York

[2] Young, P. (1984) Recursive Estimation and Time-Series Analysis. Springer-Verlag, Berlin. https://doi.org/10.1007/978-3-642-82336-7

[3] Gremal, M.S. and Andrews, A.P. (1993) Kalman Filtering-Theory and Practice. Prentice-Hall, New Jersey.

[4] Kalman, R.E. (1960) A New Approach to Linear Filtering and Prediction Problems. ASME, Series D, Journal of Basic Engineering, 82, 35-45. https://doi.org/10.1115/1.3662552

[5] Kalman, R.E. and Buch, R.S. (1961) New Results in Linear Filtering and Prediction Theory. Transactions on ASME, Series D, Journal of Basic Engineering, 83, 95-108. https://doi.org/10.1115/1.3658902

[6] Kushner, H.J. (1967) Approximations to Optimal Nonlinear Filter. IEEE Transactions on Automatic Control, 12, 546-556.

https://doi.org/10.1109/TAC.1967.1098671

[7] Bell, B.M. and Cathey, F.W. (1993) The Iterated Kalman Filter Update as a Gauss-Newton Methods. IEEE Transactions on Automatic Control, 38, 294-297. https://doi.org/10.1109/9.250476

[8] Nishiyama, K. (1997) A Nonlinear Filter for Estimating a Sinusoidal Signal and Its Parameter: On the Case of a Single Sinusoid. IEEE Transactions on Signal Processing, 45, 970-981. https://doi.org/10.1109/78.564185

[9] Vincent, T.L. and Khargonekar, P.P. (1999) A Class of Nonlinear Filtering Problems Arising from Drift Sensor Gains. IEEE Transactions on Automatic Control, 44, 
509-520.

[10] Julier, S.J. (2002) The Scaled Unscented Transformation. Proceedings of the American Control Conference, 6, 4555-4559. https://doi.org/10.1109/ACC.2002.1025369

[11] Ohta, M. and Yamada, H. (1984) New Methodological Trials of Dynamical State Estimation for the Noise and Vibration Environmental System-Establishment of General Theory and Its Application to Urban Noise Problems. Acustica, 55, 199-212.

[12] Ikuta, A. and Ohta, M. (1992) A State Estimation Method of Impulsive Signal Using Digital Filter under the Existence of External Noise and Its Application to Room Acoustics. IEICE Transactions on Fundamentals of Electronics, Communications and Computer Sciences, E75, 988-995.

[13] Ikuta, A., Ohta, M. and Masuike, H. (2006) A Countermeasure for an External Noise in the Measurement of Sound Environment and Its Application to the Evaluation for Traffic Noise at Main Line. IEEJ Transactions on EIS, 126, 63-71. https://doi.org/10.1541/ieejeiss.126.63

[14] Orimoto, H., Ikuta, A. and Xiao, Y. (2016) Method for Evaluating the Statistical Relationship between Sound Pressure Level and Noise Annoyance Based on a Nonlinear Time Series Regression Model and an Experiment. International Journal of Acoustics and Vibration, 21, 145-151. https://doi.org/10.20855/ijav.2016.21.2403

[15] Ikuta, A., Orimoto, H. and Gallagher, G. (2017) State Estimation for Fuzzy Sound Environment System with Finite Amplitude Fluctuation. Journal of Software Engineering and Applications, 10, 625-638. https://doi.org/10.4236/jsea.2017.107034

[16] Habbibi, B., Sayed, A.H. and Kailath, T. (1996) Linear Estimation in Krein Spaces-Parts I Theory and II Applications. IEEE Transactions on Automatic Control, 41, 18-33, 34-49. https://doi.org/10.1109/9.481605

[17] Ikuta, A., Masuike, H. and Ohta, M. (2007) State Estimation for Sound Environment System with Unknown Structure by Introducing Fuzzy Theory. The Institute of Electrical Engineers of Japan, 127, 770-777. https://doi.org/10.1541/ieejeiss.127.770

[18] Kitagawa, G. (1996) Monte Carlo Filter and Smoother for Non-Gaussian Nonlinear State Space Models. Journal of Computational and Graphical Statistics, 5, 1-25.

[19] Ikuta, A., Ohta, M. and Ogawa, H. (1997) Various Regression Characteristics with Higher Order among Light, Sound and Electromagnetic Waves Leaked from VDT-Measurement and Signal Processing in the Actual Working Environment. International Measurement Confederation, 21, 25-33. https://doi.org/10.1016/S0263-2241(97)00041-9

[20] Ikuta, A. (2016) A Bayesian Filter for Sound Environment System with Quantized Observation. INTER-NOISE, Hamburg, 21-24 August2016, 3184-3192. 